\title{
A study on the comprehensive carrying capacity and spatial optimization under the new development concept - an empirical study of Shandong province
}

\author{
Lili Rong ${ }^{1, *}$, Kanglei $\mathrm{Bi}^{1}$ \\ ${ }^{1}$ Business School, University of Jinan, Jinan, 250002 Shandong, China
}

\begin{abstract}
Scientific evaluation of the comprehensive carrying capacity of land is the condition for regional coordination and high-quality economic development. Applied entropy method and expert marking to determine weight, and adopted multi-objective comprehensive evaluation model to evaluate the comprehensive carrying capacity of land resources from 2008 to 2018 in Shandong province. The results show that the comprehensive carrying capacity level is located in the lower and intermediate critical level, which has obvious spatial differences, and carrying capacities of social economic, land resource, water resource and ecological environmental are not consistent. On this basis, according to obstacle characteristics of land comprehensive carrying capacity, puts forward the proposal for space optimization pattern.
\end{abstract}

\section{Introduction}

At the second plenary session of the Fifth Plenary Session of the 18th CPC Central Committee, General Secretary Xi Jinping put forward the development concept of innovation, coordination, green, openness and sharing, which is of great guiding significance to the high-quality development of China's economy. At present, the conflicts among population, resources and environment in China are beginning to emerge, such as the intensification of economic growth and excessive consumption of resources, economic development and destruction of ecological environment. The land is the carrier of the comprehensive system of social economy, population, resources and environment, and the quantitative measurement of its comprehensive carrying capacity is the prerequisite for achieving regional coordination and high-quality economic development.

The carrying capacity of land resources is an important indicator of land resources evaluation. British scholar William Allen (1949) first proposed the concept and calculation formula of land carrying capacity ${ }^{[1]}$; After the 1970s, the research on land resources carrying capacity began to gradually develop and deepen to the entire resource system. Since the 1980s, the research on the carrying capacity of land resources in China has developed rapidly. But the main direction of this research is still the carrying capacity of cultivated land and grain population. In the 1990 s, the research on the carrying capacity took into account the connection between land resources and the overall system of economic society and regional environment. For example, Niu Wenyuan (1999) gave an overall evaluation on the sustainable development capacity of 30 provinces and cities in China ${ }^{[2]}$. After entering the $21 \mathrm{st}$ century, experts and scholars paid more attention to the research on the comprehensive carrying capacity of land. For example, Wang Shuhua and Mao Hanying (2001) gave a comprehensive evaluation on the supporting systems of water and soil resources, economic technology, society and ecological environment, and then put forward an evaluation index system for the comprehensive carrying capacity of land resources ${ }^{[3]}$. Peng Wenying (2014) believe that Land resources should strengthen ecological construction and environmental protection. They put forward the theory of spatial optimization pattern of comprehensive carrying capacity of land resources ${ }^{[4]}$. Liu Lei, Zhou $\mathrm{Ce}$ and Zhang Yongfang (2016) believe that the comprehensive carrying capacity of land is an important prerequisite and guarantee for promoting regional coordination and sustainable development ${ }^{[5]}$. Many scholars have made indepth researches on both single factor bearing capacity and comprehensive bearing capacity. The analysis on that of resources among urban agglomerations which are closely related but have spatial differences is slightly less.

Based on this, the paper indicates the target layer of comprehensive carrying capacity of land resources is divided into four aspects: carrying capacity of land resources elements, carrying capacity of social and economic elements, carrying capacity of water resources elements and carrying capacity of ecological environment elements to construct corresponding evaluation index system. Entropy method is used to objectively assign weight to determine the weight of each index. With the aid of multi-objective comprehensive evaluation model, the paper explores the dynamic change rules and spatial differences of comprehensive carrying capacity in Shandong Province in the past 10 years, so as to put forward targeted and

\footnotetext{
* Corresponding author: 1 rg518@126.com
} 
differentiated suggestions for different types of land comprehensive carrying areas.

\section{Index system and model construction for measuring comprehensive carrying capacity of land resources}

\subsection{Construction of evaluation index system for land comprehensive carrying capacity}

The comprehensive carrying capacity of land is that of a certain region to all human activities under the joint support of land, population, social economy and ecological environment system. It reflects the quality and supporting capacity of land resources, social economy, water resources and ecological environment elements. The construction of the index system of land comprehensive carrying capacity needs to fully consider its connotation and system composition, combine the existing research results and the index evaluation system of land resources, and comprehensively consider the utilization status and characteristics of land resources in Shandong Province according to the scientific, systematic, data availability and application design principles of evaluation indexes. The index system of comprehensive land carrying capacity is constructed from four aspects of land resources, social economy, water resources and ecological environment, specifically including three levels of target layer, criterion layer and index layer, with a total of 16 evaluation indexes. See Table 1 for details.

\subsection{Determination of index weight}

In the evaluation of the comprehensive carrying capacity of land resources, the objective entropy method is used to determine the weight of each index. The principle of determining index weight by entropy method is determined according to the ordering degree of information contained in each index. Information entropy can describe the relative rate of sample data change. The smaller the information entropy, the more orderly the system is. On the contrary, the more chaotic the system is. Thus the index weight obtained in the comprehensive evaluation describes the relative amplitude of the index value change. Entropy method is more objective and can eliminate the interference of human subjective factors than other methods such as expert scoring, and the evaluation results are more objective and scientific. On the criterion level, there are great differences in resource endowments and economic and social development in different regions. The expert scoring method can fully combine the local conditions and characteristics, so the combination of expert scoring and entropy method is used to determine the weight of each criterion level. According to the expert consultation results, the weight order of the four criteria layer bearing systems is: land resources bearing capacity $>$ social and economic resources bearing capacity $>$ water resources bearing capacity and ecological environment bearing capacity. The calculated criteria layer and the weight coefficient results of each index are shown in table 1 .

\subsection{Evaluation model of comprehensive carrying capacity of land resources}

The comprehensive carrying capacity of land resources is a multi-objective and multi-index complex system. Therefore, the multi-objective comprehensive evaluation model is more suitable for calculating the comprehensive carrying capacity index of land resources.

According to the entropy method to determine the weight of each index, calculate the single index evaluation score formula is:

$$
S_{i j}=W_{i} \times X_{i j}
$$

Evaluation score of bearing capacity of each criterion layer:

$$
B=\sum_{i=1}^{n} S_{i j} \times W_{i}
$$

Due to the irreplaceable importance of each criterion layer, the comprehensive bearing capacity evaluation score is the sum of the scores of each criterion layer. The evaluation score of comprehensive bearing capacity index is between 0 and 1 , and the score tends to 1 , indicating that the higher the level of comprehensive bearing capacity of land resources is, the lower it is.

\subsection{Data sources and processing}

The research data mainly come from "The Statistical Yearbook of Shandong Province", "The Water Resources Bulletin of Shandong Province", "The Environment Bulletin of Shandong Province", "The Third National Land Survey" and other materials from 2008 to 2018. All kinds of land and water resources data come from the natural resources department, water resources department and field investigation of Shandong province. According to the concept of ecological land, all types of land that have the functions of maintaining regional ecological balance and providing environmental regulation belong to the category of ecological land. Combined with the existing land classification data, the area of ecological land is determined by the sum of the areas of forest land, grassland, water area and wetland. In addition, because the data of rural public green space area is not comprehensive and the number is small, the per capita public green space area index is calculated by dividing the urban public green space area by the number of urban non-agricultural population.

\section{Results and analysis}

According to the calculation method of the above evaluation model, the indexes of land resource factor bearing capacity, social and economic factor bearing capacity, water resource factor bearing capacity, ecological environment bearing capacity and land comprehensive bearing capacity of Shandong province 
and various cities are shown in Table 2. In addition, SPSS16.0 statistical analysis software is used to carry out clustering analysis on the comprehensive carrying of land by means of average connection (inter-group) clustering method. The classification tree diagram is shown in Fig. 1.

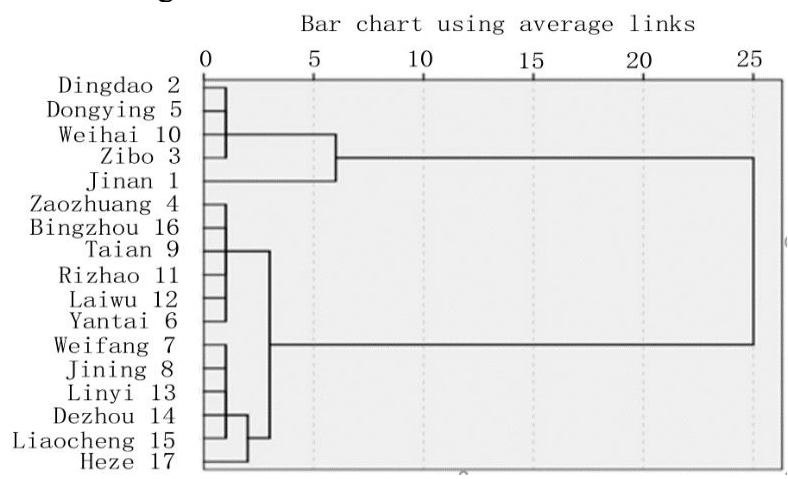

Fig. 1. Cluster analysis tree of land comprehensive carrying capacity

\subsection{The comprehensive carrying capacity of land resources is at a lower and intermediate critical level}

The evaluation score of the comprehensive carrying capacity of land resources in Shandong Province is 0.4042 , which is at the lower and intermediate critical level (the critical point is 0.4).Comparing the carrying capacity levels of various standard layers of land resources, social economy, water resources and ecological environment, the carrying capacity of land resources elements is 0.5054, which is at the intermediate level. This is because Shandong province has a high total grain production, a large population of arable land, a lower level of urbanization than regions with the same level of economic development, a higher proportion of non-agricultural population, and a larger carrying potential of construction land, resulting in a medium overall carrying capacity of land resources. The level of carrying capacity of social and economic factors is 0.5157 , which is at the intermediate level. The reason is that Shandong Province has a high GDP, abundant labor resources, high urbanization level and developed farming. Since 2012, the "strictest water resources management system" has been implemented in the whole country, and the water resources available level in Shandong province is low and the agricultural water consumption is large, which makes the carrying potential of water resources elements low, only 0.3049.The carrying capacity of ecological environment elements is 0.6747, which is at a higher level among the four element layers, mainly due to the large proportion of mountain area, large forest area and wide wetland and mudflat area located in the Yellow River basin in Shandong Province, resulting in the improvement of the overall level of ecological environment carrying capacity. The comprehensive carrying capacity of land resources in Shandong Province is at a medium level due to the combined influence of four factors: land resources, social economy, water resources and ecological environment.

\subsection{The spatial difference of land comprehensive carrying capacity of resources is significant}

The cities under the jurisdiction of Shandong Province have great differences in social and economic development level and resource endowment, and the regional differences in the comprehensive carrying capacity of land resources are obvious. According to the results of cluster analysis, Qingdao, Dongying, Weihai and Zibo are high bearing areas, Jinan is high bearing areas, Zaozhuang, Binzhou, Taian, Rizhao, Laiwu and Yantai are medium bearing areas, Weifang, Linyi, Jining, Dezhou, Liaocheng and Heze are low bearing areas.Generally speaking, there are only 5 regions with high bearing capacity and high bearing capacity, which are located in the southeast, northeast and central regions, while 12 regions with medium and low bearing capacity are concentrated in the northwest and southwest regions, which is also an important reason why the comprehensive bearing capacity of land resources in Shandong Province is at a medium level. Because of the contribution of social and economic development and the improvement of ecological environment, Qingdao, Dongying, Weihai and Zibo have the highest level of comprehensive carrying capacity of land resources, and Zibo with the highest score is at a higher level of carrying capacity. Weifang, Jining and Dezhou are restricted by water resources and ecological environment, and Heze, Linyi and Liaocheng have the lowest level of comprehensive land carrying capacity due to the limitation of social and economic development level. Heze with the lowest score is only 0.1728 . These are the areas that focus on improving the comprehensive land carrying capacity. When optimizing the allocation of land resources, the above factors need to be considered comprehensively.

\subsection{Social economy is inconsistent with the carrying capacity of land resources, water resources and ecological environment.}

On the whole, the carrying capacity of land resources in Shandong Province is basically the same as the level of social and economic carrying capacity. Comparing the carrying capacity level of land resources, social economy, water resources and ecological environment in a single region, the carrying capacity level of the four elements shows great difference, which is due to the low carrying capacity level of water resources and ecological environment in regions with high social and economic carrying capacity, high urbanization level and large population density.

\section{Suggestions on improving spatial optimization pattern of comprehensive carrying capacity of land resources}


Table 1. Evaluation index system and weight of comprehensive carrying capacity of land resources

\begin{tabular}{|c|c|c|c|c|c|}
\hline $\begin{array}{r}\text { Target } \\
\text { layer A }\end{array}$ & Criterion layer B & $\begin{array}{l}\text { Criterion } \\
\text { layer weight }\end{array}$ & Specific indicators & $\begin{array}{l}\text { Index } \\
\text { property }\end{array}$ & $\begin{array}{c}\text { Index layer } \\
\text { weight }\end{array}$ \\
\hline \multirow{16}{*}{$\begin{array}{l}\text { Compreh } \\
\text { ensive } \\
\text { carrying } \\
\text { capacity } \\
\text { of land A }\end{array}$} & \multirow{5}{*}{$\begin{array}{c}\text { Carrying Capacity } \\
\text { of Land Resources } \\
\text { Elements }\left(\mathrm{B}_{1}\right)\end{array}$} & \multirow{5}{*}{0.3358} & Land Development Intensity $\left(\mathrm{B}_{11}\right)$ & Negative (-) & 0.0554 \\
\hline & & & $\begin{array}{l}\text { The added value of the secondary and tertiary } \\
\text { industries per land }\left(B_{12}\right)\end{array}$ & Positive $(+)$ & 0.4015 \\
\hline & & & Per capita arable land $\left(\mathrm{B}_{13}\right)$ & Positive $(+)$ & 0.3169 \\
\hline & & & Per capita construction land area $\left(\mathrm{B}_{14}\right)$ & Positive $(+)$ & 0.2262 \\
\hline & & & GDP per head( $\left.\mathrm{B}_{21}\right)$ & Positive $(+)$ & 0.5664 \\
\hline & Carrying Capacity & & Population density $\left(\mathrm{B}_{22}\right)$ & Negative (-) & 0.1938 \\
\hline & Economic Factors & 0.1436 & Proportion of land used for transportation $\left(\mathrm{B}_{23}\right)$ & Positive $(+)$ & 0.2374 \\
\hline & $\left(B_{2}\right)$ & & $\begin{array}{c}\text { Ratio of added value of secondary and tertiary } \\
\text { industries to GDP }\left(\mathrm{B}_{24}\right)\end{array}$ & Positive $(+)$ & 0.0024 \\
\hline & \multirow{4}{*}{$\begin{array}{c}\text { Carrying Capacity } \\
\text { of Social and } \\
\text { Economic Factors } \\
\left(\mathrm{B}_{3}\right)\end{array}$} & \multirow{4}{*}{0.1945} & Per capita water resources $\left(\mathrm{B}_{31}\right)$ & Positive $(+)$ & 0.2047 \\
\hline & & & GDP per unit of water consumption $\left(\mathrm{B}_{32}\right)$ & Positive $(+)$ & 0.3493 \\
\hline & & & Chemical oxygen demand of water resources $\left(\mathrm{B}_{33}\right)$ & Negative (-) & 0.2223 \\
\hline & & & Matching Degree of Water and Soil Resources $\left(\mathrm{B}_{34}\right)$ & Positive $(+)$ & 0.2168 \\
\hline & \multirow{4}{*}{$\begin{array}{c}\text { Carrying } \\
\text { Capacity of } \\
\text { Ecological } \\
\text { Environmental } \\
\text { Factors }\left(\mathrm{B}_{4}\right)\end{array}$} & \multirow{4}{*}{0.3211} & Ecological Land Area Ratio $\left(\mathrm{B}_{41}\right)$ & Positive $(+)$ & 0.1664 \\
\hline & & & Forest coverage rate $\left(\mathrm{B}_{42}\right)$ & Positive $(+)$ & 0.1623 \\
\hline & & & Carbon Emission Per Unit GDP (B43) & Negative (-) & 0.1556 \\
\hline & & & Per capita public green area $\left(B_{44}\right)$ & Positive $(+)$ & 0.5157 \\
\hline
\end{tabular}

Table 2. Evaluation results of land comprehensive carrying capacity in shandong province and various cities

\begin{tabular}{|c|c|c|c|c|c|}
\hline Area & $\begin{array}{c}\text { Carrying Capacity } \\
\text { of } \\
\text { Land Resources } \\
\text { Elements } B_{1}\end{array}$ & $\begin{array}{c}\text { Carrying Capacity } \\
\text { of Social and } \\
\text { Economic Factors } \\
\mathrm{B}_{2}\end{array}$ & $\begin{array}{c}\text { Carrying Capacity } \\
\text { of Social and } \\
\text { Economic Factors } \\
\mathrm{B}_{3}\end{array}$ & $\begin{array}{c}\text { Carrying } \\
\text { Capacity of Ecological } \\
\text { Environmental Factors } \\
\mathrm{B}_{4}\end{array}$ & $\begin{array}{l}\text { Comprehensive } \\
\text { carrying capacity } \\
\text { of land A }\end{array}$ \\
\hline Shandong province & 0.5054 & 0.5157 & 0.3049 & 0.6747 & 0.4042 \\
\hline Jinan city & 0.3896 & 0.3233 & 0.4242 & 0.5460 & 0.4351 \\
\hline Qingdao city & 0.5143 & 0.5475 & 0.4386 & 0.7336 & 0.5722 \\
\hline Zibo city & 0.4576 & 0.4222 & 0.4837 & 0.9391 & 0.6099 \\
\hline Zaozhuang city & 0.2305 & 0.212 & 0.4145 & 0.3225 & 0.2920 \\
\hline Dongying city & 0.8090 & 0.9996 & 0.4127 & 0.2355 & 0.5711 \\
\hline Yantai city & 0.2346 & 0.4641 & 0.4815 & 0.3171 & 0.3409 \\
\hline Weifang city & 0.2745 & 0.2777 & 0.1742 & 0.2093 & 0.2331 \\
\hline Jining city & 0.2107 & 0.2210 & 0.3508 & 0.2069 & 0.2371 \\
\hline Taian City & 0.2933 & 0.2084 & 0.2968 & 0.2850 & 0.2776 \\
\hline Weihai city & 0.6331 & 0.4699 & 0.7762 & 0.3616 & 0.5471 \\
\hline Rizhao city & 0.3738 & 0.2975 & 0.4058 & 0.2337 & 0.3222 \\
\hline Laiwu city & 0.2455 & 0.2736 & 0.3847 & 0.3569 & 0.3112 \\
\hline linyi city & 0.2234 & 0.1989 & 0.2063 & 0.2652 & 0.2289 \\
\hline Dezhou city & 0.2930 & 0.2561 & 0.2538 & 0.2193 & 0.2549 \\
\hline Liaocheng city & 0.2220 & 0.1447 & 0.3055 & 0.1742 & 0.2107 \\
\hline Binzhou city & 0.4018 & 0.3776 & 0.284 & 0.1766 & 0.3013 \\
\hline Heze city & 0.1300 & 0.0974 & 0.3270 & 0.160 & 0.1728 \\
\hline
\end{tabular}


Based on the evaluation of the comprehensive carrying capacity of land resources and the empirical analysis of Shandong Province, the corresponding suggestions are put forward according to the spatial pattern characteristics of land resources, social economy, water resources and ecological environment.

\subsection{Spatial pattern characteristics of comprehensive carrying capacity of land resources}

4.1.1 The level of comprehensive carrying capacity of land resources in Shandong Province is relatively low, among which the level of ecological environment carrying capacity is relatively high, that of water is the lowest, and that of land and socio-economic carrying capacity is at a medium level. It is found that the low level of water resources carrying capacity is an obstacle to the low level of land resources comprehensive carrying capacity, which needs to be considered from the aspects of strengthening water resources management and improving water resources supporting capacity.

4.1.2 The comprehensive carrying capacity of land resources in Shandong Province shows a spatial differentiation characteristic of high in the east and low in the west. Among them, the comprehensive carrying capacity of land in the southeast, northeast and central regions is higher, while the carrying capacity in the northwest and southwest regions is lower. It is necessary to adjust measures to local conditions, combine the main function orientation, comprehensively consider the carrying potential of regional land resources, social economy, water resources and ecological environment, adjust and optimize the spatial utilization structure of land resources, and promote regional coordination.

4.1.3 There are great differences among the carrying capacity levels of the four elements in a single region. In order to implement sustainable development and realize regional coordinated development, each region should comprehensively consider the different carrying capacity levels and potentials of land, social economy, water resources and ecological environment to develop regional land resources and local economy on the premise of ensuring the safety and sustainability of water resources and ecological environment.

\subsection{Suggestions on improving spatial optimization pattern of comprehensive carrying capacity of land resources}

\subsubsection{Introduce price mechanism to improve water use efficiency and water resources carrying capacity}

One of the factors causing the low level of water resources carrying capacity is that as a big agricultural province, and agricultural water consumption is large and waste is serious. Second, the chemical oxygen demand put in to control water pollution is high. Improving the carrying capacity of water resources needs to be implemented by two methods: total amount control and quality control. Total amount control can start from the angle of agricultural water saving, encourage agricultural water users to take water saving measures, use the water market to sell the surplus agricultural water at a suitable price to industrial, commercial and other departments that are short of water resources, and obtain income through transfer to compensate for the cost of water saving. Let the price mechanism play the role of regulating the allocation of water resources, and realize the flow of water resources from the agricultural sector to the industrial sector, is an effective means to improve the allocation and utilization efficiency of water resources. Because once the water quality is polluted, it needs to be treated at a high cost, so the original water resource management mode of pollution before treatment needs to be changed. Specifically, the most stringent water resources management system can be implemented to prevent water pollution from the source of water, to systematically evaluate the environmental impact of water resources development projects, to strictly implement the water use approval system and to strictly punish the behavior of polluted water, so as to strengthen the sewage permit system.

\subsubsection{Innovate ideas and optimize the spatial pattern of land resources according to the main function orientation}

Overall consideration is given to the supporting conditions for scientific development and resources and environment carrying capacity in various regions. According to five new development concepts, namely, the development concepts of suitability of natural conditions, development of main functions, consideration of resources and environment carrying capacity, control of development intensity and adjustment of spatial structure, adjustment and optimization of spatial structure of land resources are carried out according to local conditions to realize coordinated and sustainable development among regions. According to the regional resources and environment carrying capacity, current development intensity and future carrying potential, different development forms are determined. The national land resources development space in Shandong province is divided into urbanization area, agricultural product supply function area and ecological environment fragile protection area according to the different main products provided by each region.

Urbanization area. Urbanized areas defined by the content of land development include optimized development areas and key development areas. Jinan, Qingdao, Yantai, Weihai, Dongying and Zibo have high level of economic carrying capacity, strong comprehensive strength and economic foundation. They are population and economic gathering areas, with industrial products and service products as the main functions, and belong to the optimized development area. In addition, Weifang and Rizhao have high carrying capacity of construction land, which is a key development area. The above-mentioned areas are optimized and focused on development. The direction of 
land use is ensuring the functions of basic and public service facilities, further intensively and efficiently utilize construction land, and maintain the balance between social and economic development, production and ecological land. At the same time, through land use planning, industrial structure adjustment and integration of various types of land, an efficient urban functional area with reasonable urban layout and optimized industrial allocation is formed. It will become a leading area to support and lead the economic development of the whole province and lead the transformation and upgrading of economic structure.

Agricultural product supply functional area. Binzhou, Weifang, Dezhou, Tai an, Liaocheng, Jining, Zaozhuang, Heze and Dongying are rich in cultivated land resources and have good agricultural production conditions. They are the main producing areas for agricultural products. They are of great significance for ensuring food security in the whole province and even in the whole country. They are areas that focus on maintaining and improving the production and supply capacity of agricultural products. The direction of land use in this region is to strictly implement the national basic farmland protection system, protect high-quality farmland, transform and enhance the output capacity of medium and low-yield farmland, speed up farmland consolidation and land reclamation, and provide the necessary land resources for food security.

Protected areas with fragile ecological environment. Dezhou, Liaocheng and Heze regions have the lowest carrying capacity of ecological environment and belong to regions with fragile ecological environment.As an important grain production area, land resources are abundant, but the population is large and the ecological environment is poor. On the premise of maintaining agricultural production, land ecological environment should be continuously improved and wasteland and barren hills reclaimed. From an economic and social point of view, the economic society and urban construction in these regions are generally at a low level, and the level of urban public greening construction is low. In urban planning, attention is paid to the ecological services and landscaping functions of land to ensure the necessary supply of ecological land. In addition, the industries in these regions are mostly low-end processing and manufacturing industries, which have serious pollution to the atmospheric environment and high carbon emissions per unit of GDP. The balance between agricultural production, development of emerging industries and ecological livable construction should be achieved by gradually eliminating high energy consuming and polluting enterprises and focusing on the introduction of emerging environmental protection industries.

Fund Project: National Social Science Project "research on the large-scale transaction and its effect of right-tohousehold agricultural water right" (18BJY075)

\section{References}

1. W. A. Allan, Studies in African land usage in Northern Rhodesia, Rhodes Livingstone Papers and No.15. Cape Town: Oxford University Press (1949)

2. W. Y. Niu, Sustainable Development: An Inevitable Choice for China's Strategic Development in the 21st century. J. Forum on Science and Technology in China. 22 (5): 13-15 (1999)

3. S. H. Wang, H. Y. Mao, Design and evaluation on the indicator system of land comprehensive carrying capacity. J. Journal of Natural Resources. 16 (3):248-254 (2001)

4. W. Y. Peng, N. B. Liu, L. Y. Zhang, Land Resources' Synthetic Carrying Capacity and the Spatial Optimized Pattern of China's Capital Circle. J. Journal of capital university of economics and business. 16(1):77-83 (2014) 5. L. Liu, C. Zhou, Y. F. Zhang, Study on Regional Differentiation of Land Comprehensive Carrying Capacity from the Perspective of Tianjin-Hebei Coordinated Development. J. Social Sciences in Guangxi. 251 (5):84-89 (2016)

6. C. F. Yang, et al, Spatial agglomeration and its influential factors of Shandong Province's county-level floating popultion. J. Urban Issues. 289 (8):61-69 (2019) 